\title{
Predictive significance of STK17A in patients with gastric cancer and association with gastric cancer cell proliferation and migration
}

\author{
ZEHUA WANG $^{1}$, CHENYI WANG $^{2}$, BING-HUA JIANG ${ }^{3}$, LITONG SHI $^{1}$, SHAN LIN $^{1}$, \\ LEI WANG ${ }^{1}$, LING-ZHI LIU ${ }^{3}$, JIAN-GE QIU ${ }^{3}$, YANRU QIN ${ }^{1}$ and YONGXU JIA ${ }^{1}$ \\ Departments of ${ }^{1}$ Oncology and ${ }^{2}$ General Surgery, The First Affiliated Hospital of Zhengzhou University; \\ ${ }^{3}$ Academy of Medical Science, Zhengzhou University, Zhengzhou, Henan 450052, P.R. China
}

Received November 17, 2020; Accepted April 12, 2021

DOI: $10.3892 /$ or.2021.8070

\begin{abstract}
Gastric cancer (GC) is one of the most frequently diagnosed types of cancer worldwide, and exploring its potential therapeutic targets is particularly important for improving the prognosis of patients with GC. The aim of the present study was to investigate the association between serine/threonine kinase 17a (STK17A) expression and GC prognosis. STK17A expression was measured by quantitative real-time PCR, western blotting and immunohistochemical staining. Standard stable transfection technology was also used to construct overexpression and knockdown cell lines. Wound healing, Transwell, Cell Counting Kit-8 and colony formation assays, as well as other methods, were used to explore the function and underlying molecular mechanism of STK17A in GC. The results indicated that STK17A overexpression significantly promoted the proliferation and migration of GC cells. The clinical significance of STK17A in a cohort of 102 cases of GC was assessed by clinical correlation and Kaplan-Meier analyses. Overexpression of STK17A was demonstrated to be associated with tumor invasion depth $(\mathrm{P}<0.001)$, lymph node metastasis $(\mathrm{P}<0.001)$ and poor prognosis in terms of 5 -year survival $(\mathrm{P}<0.001)$. In addition, Cox multivariate analysis revealed that STK17A expression was an independent risk factor for overall and progress-free survival $(\mathrm{P}<0.001)$. Therefore, STK17A may be a valuable biomarker for the prognosis of patients with GC.
\end{abstract}

Correspondence to: Dr Yanru Qin or Dr Yongxu Jia, Department of Oncology, The First Affiliated Hospital of Zhengzhou University, 1 Jian She East Road, Erqi, Zhengzhou, Henan 450052, P.R. China E-mail: yanruqin@163.com

E-mail: jiayongxu111@126.com

Key words: serine/threonine kinase 17a, gastric cancer, prognosis, proliferation, migration

\section{Introduction}

Gastric cancer (GC) is one of the most frequently diagnosed types of cancer worldwide, with an estimated 1,089,103 newly diagnosed cases and 768,793 deaths worldwide in 2020 (1). Although there are numerous different treatments for GC, the prognosis of patients with advanced GC remains poor (2). Lymph node invasion and distant metastasis are the main factors that result in death and poor prognosis (3). Therefore, it is urgent to identify novel diagnostic and prognostic biomarkers, which could contribute to improved outcomes in patients with GC.

Serine/threonine kinase 17a (STK17A; also known as death-associated protein kinase-related apoptosis-inducing protein kinase 1; DRAK1) is a serine/threonine kinase. STK17A is a member of the death-associated protein kinase (DAPK) family and acts as a positive regulator of apoptosis $(4,5)$. STK17A was recently found to serve an important role in cell proliferation, apoptosis, tumor metastasis and tumorigenesis (6-11). However, the association between STK17A expression and the development of GC remains unclear.

At present, research on the molecular function of the DAPK family is mainly focused on the interaction between phosphorylation of the myosin light chain (MLC) regulatory subunit and the cytoskeleton, which leads to the regulation of actin stress fibers, adhesion junctions and cell movement (12-14). Recent genetic evidence indicates that DRAKs are involved in the regulation of cell shape and adhesion and cytoskeletal dynamics through phosphorylation of the Drosophila ortholog of MLC (15-17). MLC has been identified as one of the few targets for STK17A phosphorylation (4). A study on STK17A indicated that the expression of STK17A induced apoptosis-related morphological changes associated with membrane blistering, which was also induced by MLC phosphorylation $(4,18)$. In addition, STK17A affects cell contractility by regulating weak metabolism, which further promotes metastasis and spread. Weak metabolism is a type of cell death triggered by cell detachment, which is necessary for survival in the lymphatic and vascular systems (19). This indicates a new direction for exploring the relationship between STK17A and tumor invasion, progression and metastasis. 
In recent years, research on the association between STK17A and chemotherapy resistance has also yielded important results. It has been demonstrated that STK17A exhibits low expression in acquired drug-resistant cell phenotypes, which are resistant to oxaliplatin and 5-fluorouracil (6). In MeWo cells, a malignant melanoma cell line, STK17A was also revealed to be associated with cross-resistance to DNA-damaging drugs (20). These results indicated that STK17A may mediate the response of tumor cells to chemotherapy and may be important for the development of cancer chemotherapeutics.

The aim of the present study was to investigate the STK17A mRNA and protein expression in GC cells and human GC tissues, and determine the effects of STK17A overexpression and silencing on the proliferation and migration of GC cells. Furthermore, its association with clinicopathological characteristics and its significance for the prognosis of GC were explored, in order to determine whether STK17A may be of value as a prognostic factor and potential therapeutic target for GC.

\section{Materials and methods}

Clinical samples. A total of $39 \mathrm{GC}$ and matched normal tissue samples were obtained from patients with GC (aged from 41 to 76 years) who underwent surgical excision at the First Affiliated Hospital of Zhengzhou University (Zhengzhou, China) between October 2015 and March 2016, and were used for RNA extraction. Of the 39 samples, 26 were male and 13 were female. The inclusion criteria were as follows: Definite diagnosis of primary gastric cancer; successful radical resection of gastric cancer and $\mathrm{R} 0$ resection. The exclusion criteria were as fllows: Malignant tumors with other tissue origins; death from causes other than gastric cancer. A total of 102 formalin-fixed paraffin-embedded GC samples from the period between January 2008 and December 2010 were also collected from the same hospital and STK17A immunohistochemistry was performed on all samples. No patients had undergone chemotherapy or radiotherapy prior to surgical resection. In addition, tumors were staged according to the TNM staging criteria of the American Joint Committee on Cancer (7th edition, 2010) (21). Written informed consent for the use of tissue samples was obtained on their behalf from all from all patients or their legal guardians. The use of GC tissues in the present study was approved by the Ethics Committee of Zhengzhou University.

Cell lines. Human GC cell lines used in this study included AGS (RPMI-1640), HGC27 (RPMI-1640), KATOIII (IMDM), MKN45 (RPMI-1640), NCI-N87 (RPMI-1640), and SNU1 (RPMI-1640), normal gastric cell GES-1 (RPMI-1640), and 293T (DMEM) were obtained from the Cell Bank of Type Culture Collection of Chinese Academy of Sciences. All culture media were obtained from GIBCO BRL Life Technologies. All cells were cultured in medium supplemented with $10 \%$ FBS and 1\% penicillin-streptomycin (all from Gibco; Thermo Fisher Scientific, Inc.) at $37^{\circ} \mathrm{C}$ in humidified air with $5 \% \mathrm{CO}_{2}$.

Reverse transcription-quantitative $(R T-q) P C R$ analysis. Fresh tissue samples were immediately frozen in liquid nitrogen after surgical resection and then stored at $-80^{\circ} \mathrm{C}$
Table I. Primer sequences of STK17A and GAPDH.

\begin{tabular}{ll}
\hline Gene & \multicolumn{1}{c}{ Sequences } \\
\hline STK17A & q-F: 5'-TCTGAGTCGGCTGTTGATTTC-3' \\
& q-R: 5'-GGGGTGCTTTAGACATTCTTCA-3' \\
GAPDH & q-F: 5'-GCTGAACGGGAAGCTCACTG-3' \\
& q-R: 5'-GTGCTCAGTGTAGCCCAGGA-3' \\
\hline
\end{tabular}

STK17A, serine/threonine kinase 17a; q, qPCR; F, forward; R, reverse.

prior to RNA isolation. Total RNA was extracted from tissue samples with TRIzol ${ }^{\circledR}$ reagent (Thermo Fisher Scientific, Inc.) and reverse-transcribed to cDNA using the Reverse Transcription for PCR Kit (Takara Bio, Inc.) according to the standard protocols provided by the manufacturer. qPCR was performed with the ABI7900HT Fast Real-Time PCR system (Applied Biosystems; Thermo Fisher Scientific, Inc.) using a SYBR Green PCR Kit (Applied Biosystems; Thermo Fisher Scientific, Inc.). The primer sequences used for STK17A and GAPDH are presented in Table I. The following PCR conditions were used: Initial denaturation at $95^{\circ} \mathrm{C}$ for $2 \mathrm{~min}$, followed by 40 cycles of denaturation at $94^{\circ} \mathrm{C}$ for $15 \mathrm{sec}$, and annealing/extension at $60^{\circ} \mathrm{C}$ for $1 \mathrm{~min}$. The relative expression of STK17A mRNA against GAPDH was calculated using the $2^{-\Delta \Delta \mathrm{Cq}}$ method (22).

Tissue microarray (TMA) and immunohistochemical staining. Using 102 pairs of GC tissue samples and corresponding normal tissues, TMAs were constructed as previously described (23). The archived paraffin-embedded tissue blocks (21) were cut into $4-\mu \mathrm{m}$ sections on adhesion slides and then sections were dried in a thermostat oven for $2 \mathrm{~h}$ at $65^{\circ} \mathrm{C}$. Immunocytochemical staining was performed using the streptavidin-biotin complex method to investigate the expression of STK17A. In brief, tissue sections were deparaffinized in xylene and rehydrated in ethanol, followed by antigen retrieval in $0.1 \mathrm{~mol} / 1$ citrate buffer solution ( $\mathrm{pH}$ 6.0) in a microwave oven for $\sim 15 \mathrm{~min}$. After washing in PBS, the sections were blocked in $10 \%$ goat serum for $10 \mathrm{~min}$ at room temperature to eliminate nonspecific binding. The sections were then incubated overnight with primary anti-STK17A polyclonal antibody (1:150 dilution; ab97530, Abcam) at $4^{\circ} \mathrm{C}$ in humidity. In the next step, the sections were sequentially incubated with a biotin-labeled goat anti-rabbit IgG (cat. no. SP-9000; Beijing Zhongshan Golden Bridge Biotechnology, Co., Ltd.) at a concentration of 1:100, at room temperature for $30 \mathrm{~min}$. Finally, diaminobenzidine (Beijing Zhongshan Golden Bridge Biotechnology, Co., Ltd.) was used for color development for $1 \mathrm{~min}$ at room temperature, followed by hematoxylin (Beijing Zhongshan Golden Bridge Biotechnology, Co., Ltd.). counterstaining for $2 \mathrm{~min}$ at room temperature.

Two independent pathologists evaluated the immunohistochemical results in a blinded manner. STK17A-positive staining intensity was scored as follows: 0 , negative; 1 , weak; 2 , moderate; and 3 , strong. In addition, the percentage of STK17A-positive cells was scored as follows: $0,<5 ; 1,5-25$; $2,26-50 ; 3,51-75$; and $4,>75 \%$. The final staining scores were 

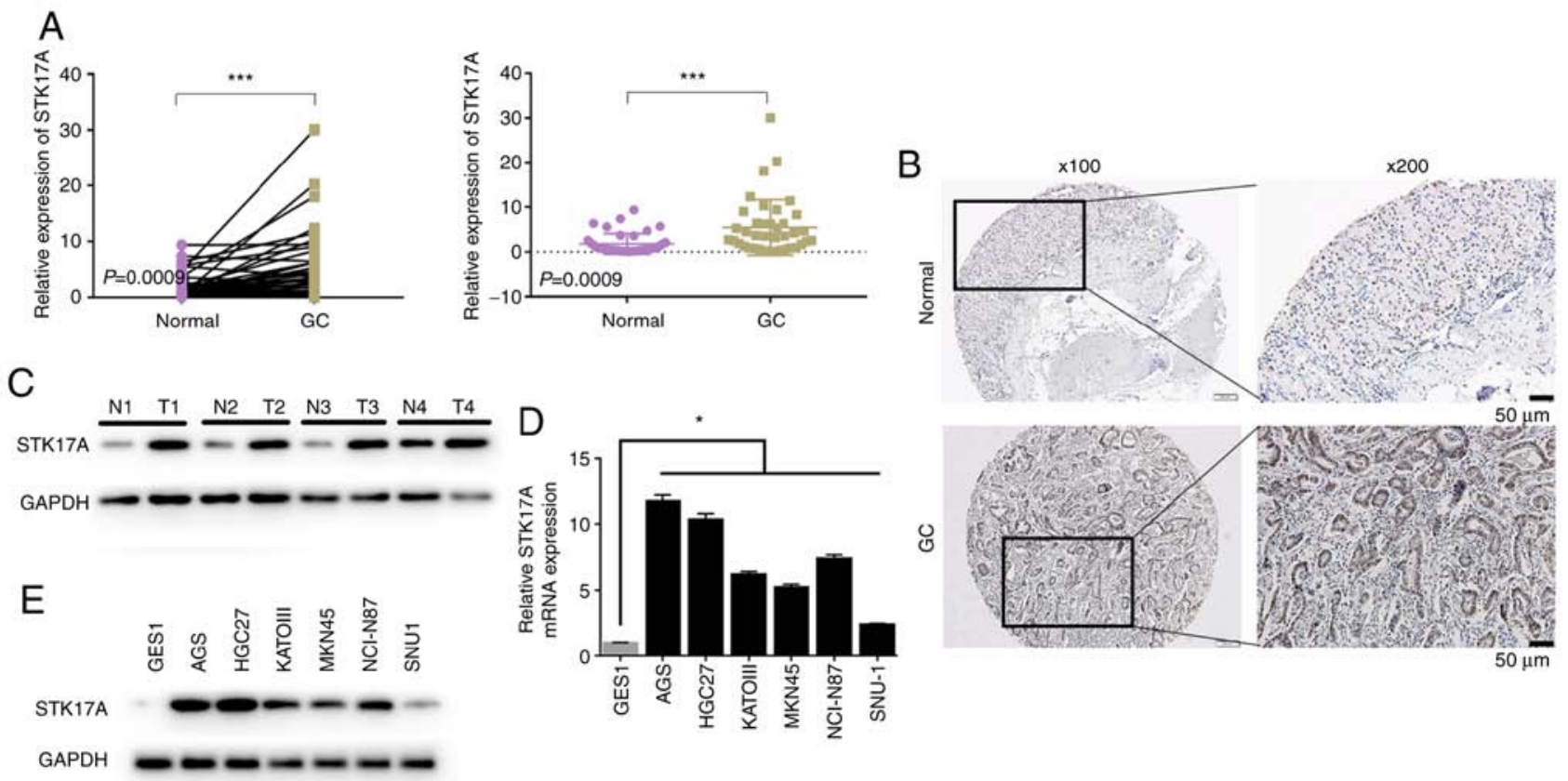

Figure 1. STK17A is upregulated in GC. (A) GC tissues have an increased level of STK17A mRNA than their paired adjacent tissues. ${ }^{* * *} \mathrm{P}=0.0009$, paired t-test. (B) The sections were studied using a light microscope (Olympus BX 53 light microscope; magnifications, $\mathrm{x} 100$ and x200; and scale bar, $50 \mu \mathrm{m}$ ) (C) STK17A protein expression was markedly increased in gastric tumor tissues than their adjacent normal tissues. (D) Real-time PCR analysis of STK17A expression in normal gastric epithelial GES1 cells and GC cell lines. ${ }^{*} \mathrm{P}<0.05$. (E) Western blotting of STK17A expression in GES1 and GC cell lines. STK17A, serine/threonine kinase 17a; GC, gastric cancer.

calculated by multiplying the scores for the percentage of STK17A-positive cells and staining intensity. To divide patients into high- and normal-expression groups, the cutoff value was determined by receiver operating characteristic curve analysis. The sections were studied using a light microscope (Olympus BX 53; Olympus Corporation).

Western blot analysis. Total proteins were extracted from GC tissues and tumor cells using ice-cold RIPA lysis buffer (Thermo Fisher Scientific, Inc.). The protein concentration was determined using a bicinchoninic acid protein assay kit. Proteins $(20 \mu \mathrm{g})$ were heated in loading buffer at $100^{\circ} \mathrm{C}$ for $10 \mathrm{~min}$ and separated by $10 \%$ SDS-PAGE, followed by transfer onto PVDF membranes. After blocking with $5 \%$ skimmed milk at room temperature for $1 \mathrm{~h}$, the membranes were probed at $4^{\circ} \mathrm{C}$ overnight with primary antibodies against $\mathrm{N}$-cadherin (1:1,000 dilution; product no. 13116), E-cadherin (1:1,000 dilution; product no. 3195), vimentin (1:1,000 dilution; product no. 5741) and GAPDH (1:1,000 dilution; product no. 5174; all from Cell Signaling Technology, Inc.) and STK17A (1:1,000 dilution; Abcam), After washing in TBST three times, the blots were incubated with the HRP-conjugated secondary antibodies (anti-rabbit IgG; 1:8,000 dilution, product no. 7074; Cell Signaling Technology, Inc.) at room temperature for $1 \mathrm{~h}$. Finally, an enhanced chemiluminescence detection system (Imager 600; Amersham; Cytiva) was used to quantify the protein levels with enhanced chemiluminescent reagent (ECL; Epizyme, Inc.).

Cell Counting Kit-8(CCK-8) assay. The transfected tumor cells were plated in a 96-well plate at a density of 1,000 cells/well. CCK-8 reagent (10 $\mu \mathrm{l}$; Dojindo Molecular Technologies, Inc.) was added to $100 \mu 1$ RPMI-1640 culture medium at 1-4 and 5 days and the sample was incubated in a cell culture incubator for $2 \mathrm{~h}$ at $37^{\circ} \mathrm{C}$. Absorbance was detected at $450 \mathrm{~nm}$ using a microplate reader to generate a cell proliferation curve. At least three determinations were performed in triplicate.

Colony formation assay. Tumor cells and control cells were plated in triplicate in 6-well plates at a density of 1,000 cells/well. After 7 days of culture, the cells were fixed with $4 \%$ paraformaldehyde for $30 \mathrm{~min}$ at room temperature, and then stained with $0.1 \%$ crystal violet solution for $30 \mathrm{~min}$ at room temperature. Finally, images were captured and the number of cell colonies was counted, and clones with $\geq 50$ cells were scored as actual colonies. At least three determinations were performed in triplicate.

Wound healing assay. Cells (AGS, HGC27, MKN45 and SNU1) were uniformly seeded in 6-well plates and cultured to $90 \%$ confluence. The cell monolayer was washed with PBS and a $100-\mu 1$ pipette tip was used to scrape the bottom of the plate and create linear scratches. Then, the cell culture medium was replaced with RPMI-1640 serum-free medium. After scratching, images of the wound area were captured under a light microscope (Olympus CKX 53; Olympus Corporation) at 0 and $16 \mathrm{~h}$. At least three determinations were performed in triplicate.

Transwell assay. Transwell experiments were performed using 24-well $(8.0 \mu \mathrm{M})$ Transwell inserts (BD Biosciences). The cells were resuspended in serum-free RPMI-1640 medium, and $1 \times 10^{5}$ cells were seeded into the upper chamber of a 24-well plate. RPMI-1640 medium supplemented with $10 \%$ 
Table II. Relationship between STK17A protein expression in gastric cancer tissue and its clinical pathological parameters.

\begin{tabular}{|c|c|c|c|c|}
\hline \multirow[b]{2}{*}{ Clinicopathological parameters } & \multirow[b]{2}{*}{ All cases } & \multicolumn{2}{|c|}{ STK17A expression } & \multirow[b]{2}{*}{ P-value } \\
\hline & & High & Low & \\
\hline \multicolumn{5}{|l|}{ Sex } \\
\hline Male & 79 & 41 & 38 & \multirow[t]{2}{*}{0.815} \\
\hline Female & 23 & 11 & 12 & \\
\hline \multicolumn{5}{|l|}{ Age (years) } \\
\hline$<60$ & 57 & 28 & 29 & \multirow[t]{2}{*}{0.695} \\
\hline$\geq 60$ & 45 & 24 & 21 & \\
\hline \multicolumn{5}{|l|}{ Lauren classification } \\
\hline Diffuse type & 26 & 17 & 9 & \multirow[t]{2}{*}{0.018} \\
\hline Intestinal and mixed type & 76 & 35 & 41 & \\
\hline \multicolumn{5}{|l|}{ Differentiation } \\
\hline Well and moderate & 52 & 30 & 22 & \multirow[t]{2}{*}{0.384} \\
\hline Poor & 50 & 22 & 28 & \\
\hline \multicolumn{5}{|l|}{ Tumor invasion depth } \\
\hline $\mathrm{T} 1 / \mathrm{T} 2$ & 25 & 2 & 23 & \multirow[t]{2}{*}{$<0.001$} \\
\hline $\mathrm{T} 3 / \mathrm{T} 4$ & 77 & 50 & 27 & \\
\hline \multicolumn{5}{|l|}{ Location } \\
\hline Upper & 17 & 10 & 7 & \multirow[t]{3}{*}{0.449} \\
\hline Middle & 81 & 39 & 42 & \\
\hline Lower & 4 & 3 & 1 & \\
\hline \multicolumn{5}{|l|}{ pTNM stage } \\
\hline $\mathrm{I} / \mathrm{II}$ & 49 & 9 & 40 & \multirow[t]{2}{*}{$<0.001$} \\
\hline III/IV & 53 & 43 & 10 & \\
\hline \multicolumn{5}{|l|}{ Lymph node metastasis } \\
\hline Yes & 52 & 41 & 11 & \multirow[t]{2}{*}{$<0.001$} \\
\hline No & 50 & 11 & 39 & \\
\hline \multicolumn{5}{|l|}{ Survival status } \\
\hline Survival & 35 & 5 & 30 & \multirow[t]{2}{*}{$<0.001$} \\
\hline Death & 67 & 47 & 20 & \\
\hline
\end{tabular}

STK17A, serine/threonine kinase 17a.

FBS was added to the lower chamber. After culturing the cells in an incubator containing $5 \% \mathrm{CO}_{2}$ at $37^{\circ} \mathrm{C}$ for $16 \mathrm{~h}$, the cells remaining on the surface of the membrane were removed with a cotton swab, and the migrated cells were fixed with $4 \%$ methanol solution for $30 \mathrm{~min}$ at room temperature, stained with $0.1 \%$ crystal violet solution for $30 \mathrm{~min}$ at room temperature and images were captured under a light microscope.

Transfection. PEZ-Lv201-STK17A, psi-LVRU6GP-shRNA targeting STK17A and the corresponding control plasmids were purchased from GeneCopoeia, Inc.. Lentivirus containing STK17A and shRNA was packaged using the 3rd generation ViraPower $^{\mathrm{TM}}$ Lentiviral Packaging Mix (Invitrogen; Thermo Fisher Scientific, Inc.) in 293T cells. The day before transfection, 293T cells were plated in 10-cm dishes cultured in DMEM with $10 \%$ FBS. When cell density reached $\sim 60-80 \%$, the cells were transfected with the above plasmids using Lipofectamine ${ }^{\circledR} 2000$ (Invitrogen; Thermo Fisher Scientific,
Inc.). The mass of lentiviral plasmid used was $20 \mu \mathrm{g}$ and the ratio of the lentiviral plasmid, packaging vector and envelope vector was 4:3:2. After, the transfected cells were incubated at $37^{\circ} \mathrm{C}$ in a $5 \% \mathrm{CO}_{2}$ incubator for $\sim 8-12 \mathrm{~h}$, the culture medium was replaced with $\sim 5-6 \mathrm{ml}$ fresh DMEM with $10 \%$ FBS. The supernatants containing viruses were harvested at 24 and $48 \mathrm{~h}$ and concentrated by ultracentrifugation for $90 \mathrm{~min}$ at $50,000 \times \mathrm{g}, 4^{\circ} \mathrm{C}$, and then stored at $-80^{\circ} \mathrm{C}$ until use.

Cells were infected with lentiviruses according to the protocol of the manufacturer. Briefly, cells were plated in 24-well plates at $1 \times 10^{5}$ cells/well, lentiviruses were added into culture medium separately (the volume of lentiviruses was calculated as an MOI of 20), and medium was refreshed after $12 \mathrm{~h}$. Puromycin was used to screen the stable cells after $72 \mathrm{~h}$ of lentiviral infection at $1.0 \mu \mathrm{g} / \mathrm{ml}$ as a final concentration.

Statistical analysis. SPSS 21.0 software (IBM Corp.) was used to perform statistical analysis. Paired two-tailed t-test 
Table III. Univariate Cox regression analysis for OS and PFS in gastric cancer.

\begin{tabular}{lrrrrr}
\hline & \multicolumn{2}{c}{ OS } & \multicolumn{2}{c}{ PFS } \\
\cline { 2 - 3 } Clinical parameters & HR $(95 \% \mathrm{CI})$ & P-value & & HR $(95 \% \mathrm{CI})$ & P-value \\
\hline Age & $0.629-1.656$ & 0.935 & $0.621-1.635$ & 0.974 \\
Sex & $0.676-2.086$ & 0.551 & $0.667-2.053$ & 0.583 \\
Lauren classification & $0.800-1.176$ & 0.760 & $0.823-1.207$ & 0.971 \\
Differentiation & $0.595-1.061$ & 0.120 & $0.603-1.708$ & 0.146 \\
Tumor invasion depth & $1.444-2.744$ & $<0.001$ & $1.417-2.673$ & $<0.001$ \\
pTNM stage & $1.569-3.181$ & $<0.001$ & & $1.503-3.014$ & $<0.001$ \\
Lymph node metastasis & $1.799-5.109$ & $<0.001$ & & $1.622-4.498$ & $<0.001$ \\
STK17A expression & $2.846-8.865$ & $<0.001$ & $2.573-7.619$ & $<0.001$ \\
\hline
\end{tabular}

OS, overall survival; PFS, progression-free survival; STK17A, serine/threonine kinase 17a.

Table IV. Cox multivariate regression analysis for OS and PFS in gastric cancer.

OS

PFS

\begin{tabular}{lcccr}
\cline { 2 - 3 } Parameters & HR $(95 \% \mathrm{CI})$ & P-value & HR $(95 \% \mathrm{CI})$ & P-value \\
\hline STK17A expression & $1.871-6.715$ & $<0.001$ & $1.744-5.929$ & $<0.001$ \\
pTNM stage & $1.042-2.299$ & 0.031 & $1.022-2.218$ & 0.039
\end{tabular}

OS, overall survival; PFS, progression-free survival; STK17A, serine/threonine kinase 17a.
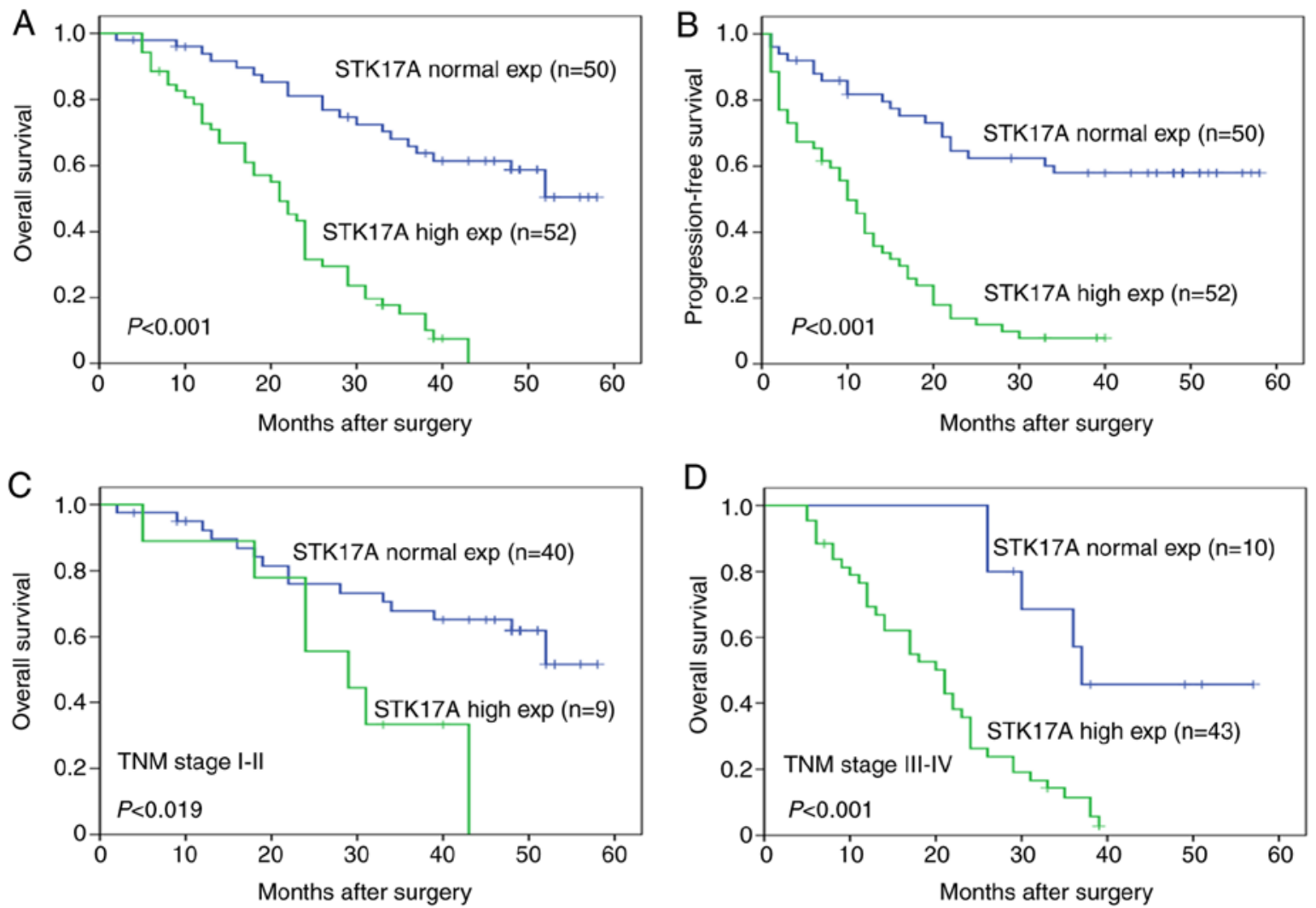

Figure 2. Survival curves plotted by employing Kaplan-Meier estimator. (A and B) Kaplan-Meier overall survival and progression-free survival curves for all 102 patients with GC stratified by high and normal expression of STK17A. (C and D) Kaplan-Meier overall survival curves for GC patients with TNM stage I-II and III-IV. STK17A, serine/threonine kinase 17a; GC, gastric cancer. 

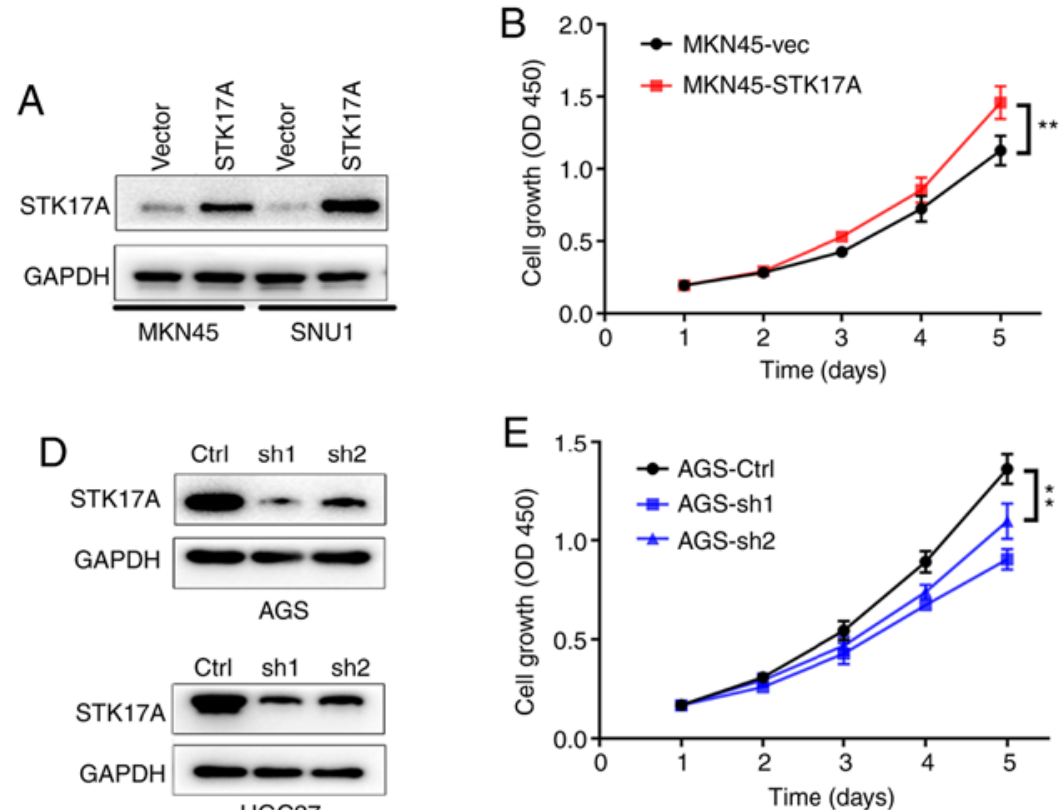

G

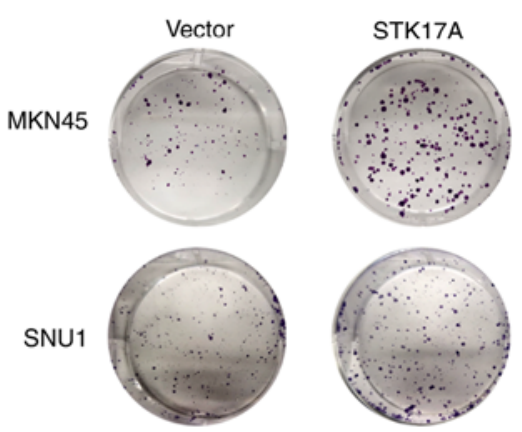

$J$
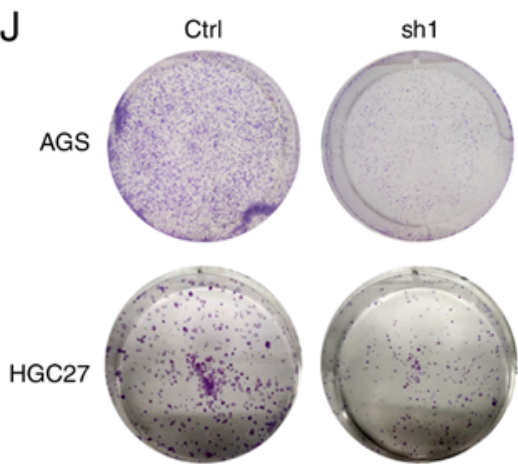
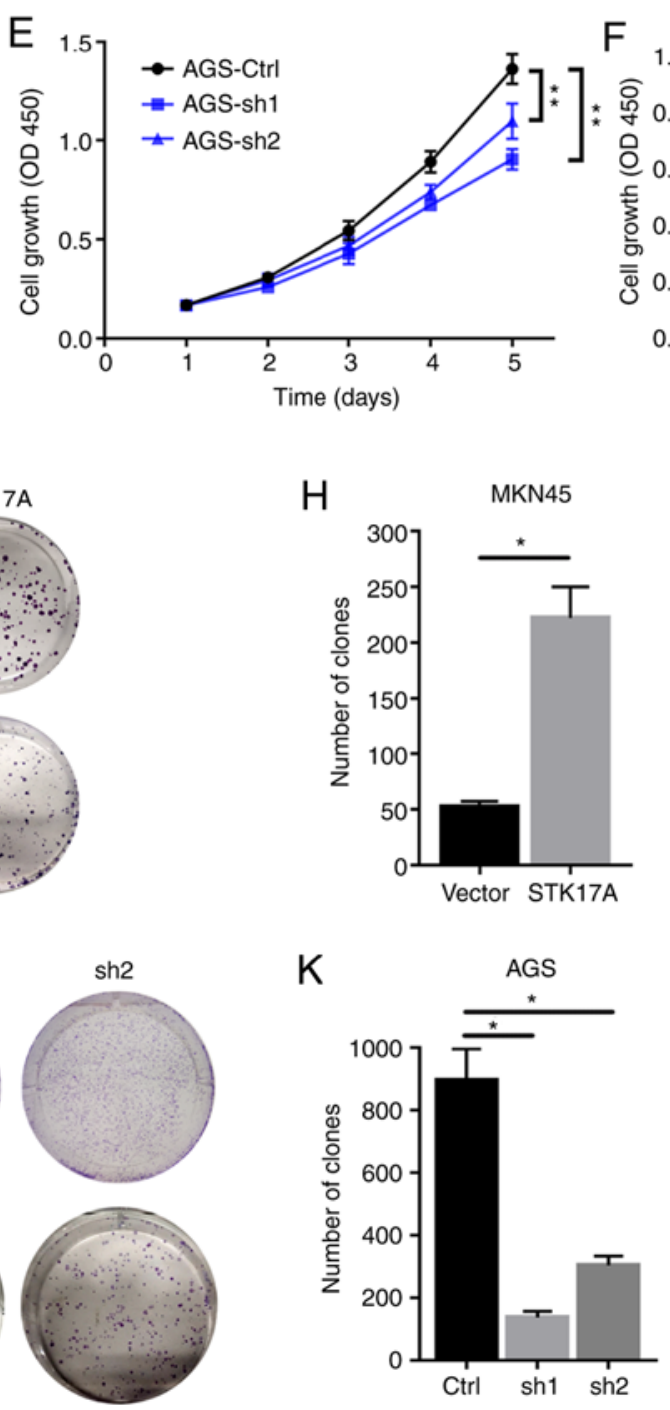

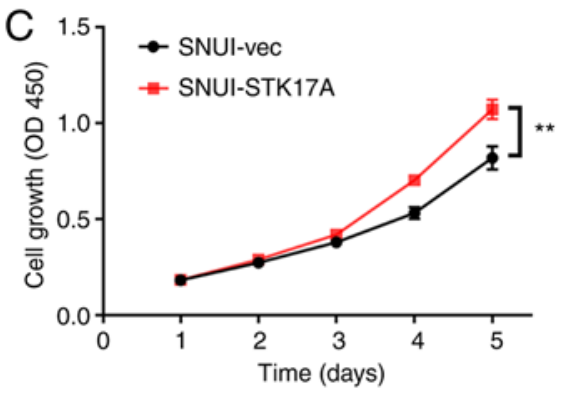

$\mathrm{F}$

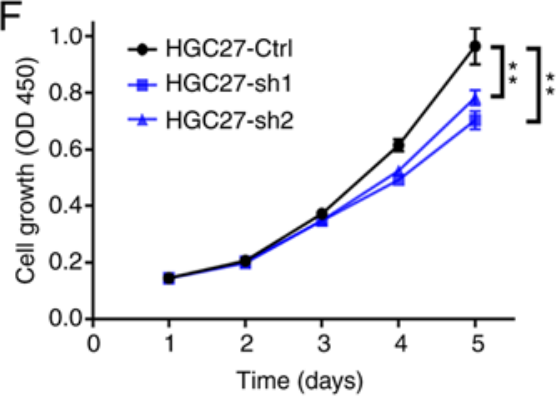

Figure 3. STK17A upregulation promotes GC cell proliferation and migration. (A) Western blotting indicated the expression of STK17A and GAPDH in transfected cells. (B and C) The effect of STK17A-overexpressed expression on GC cell growth using the CCK-8 assay. (D) Western blotting indicated the expression of STK17A and GAPDH in transfected STK17A-knockdown cells. (E and F) The effect of STK17A-knockdown on GC cell growth using the CCK-8 assay. (G-I) Overexpression of STK17A increased the mean number of colonies in the colony formation assay. (J-L) Downregulation of STK17A inhibited the mean number of colonies in the colony formation assay. ${ }^{*} \mathrm{P}<0.05$ and ${ }^{* *} \mathrm{P}<0.01$. STK17A, serine/threonine kinase $17 \mathrm{a}$; GC, gastric cancer; CCK-8, Cell Counting Kit-8; Ctrl, control; sh, short haiprin.

was performed to compare STK17A expression between cancer tissues and their paired adjacent tissues. In addition, the statistical significance of associations between STK17A protein expression and clinicopathological characteristics was analyzed using the $\chi^{2}$ test. Survival rates were determined using the Kaplan-Meier method, and survival curves were compared using log-rank testing, while the prognostic value of STK17A was analyzed with the Cox-proportional hazard model. Data from three separate experiments are presented as the mean values \pm standard deviation (SD). The Student's t-test was used for comparisons between single experimental groups and the control groups. Multigroup comparisons of the means were carried out by one-way analysis of variance (ANOVA) test, followed by Dunnett's post hoc test. $\mathrm{P}<0.05$ was considered to indicate statistically significant differences. 


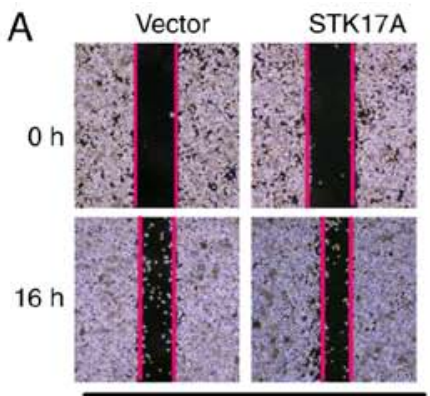

MKN45

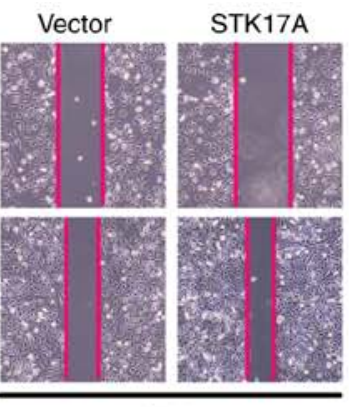

SNU1

C
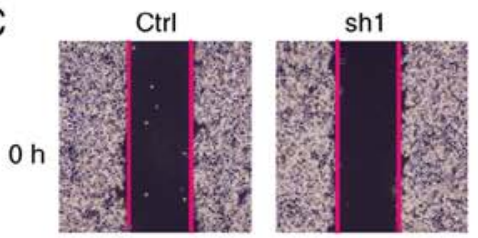

$16 \mathrm{~h}$

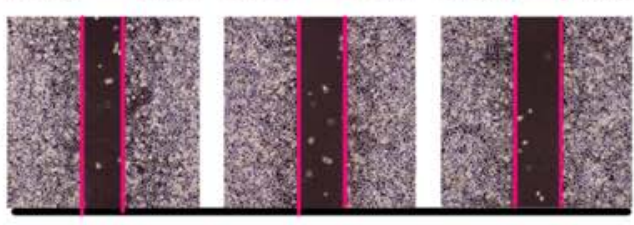

AGS

E

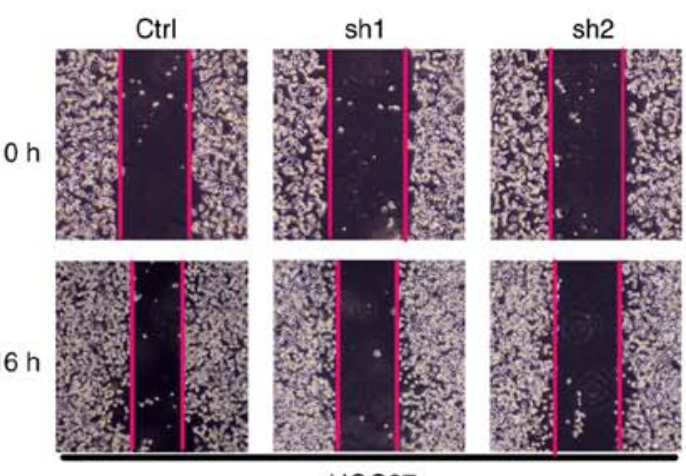

HGC27

G
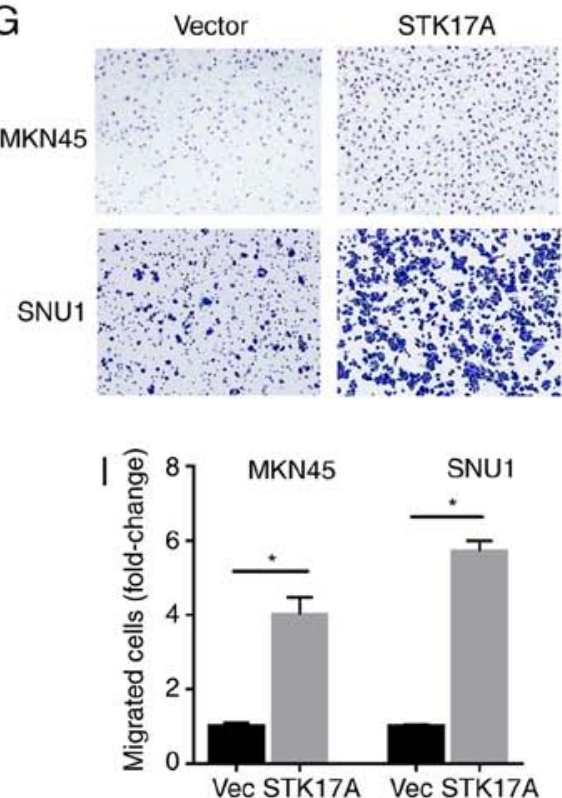

\section{B}
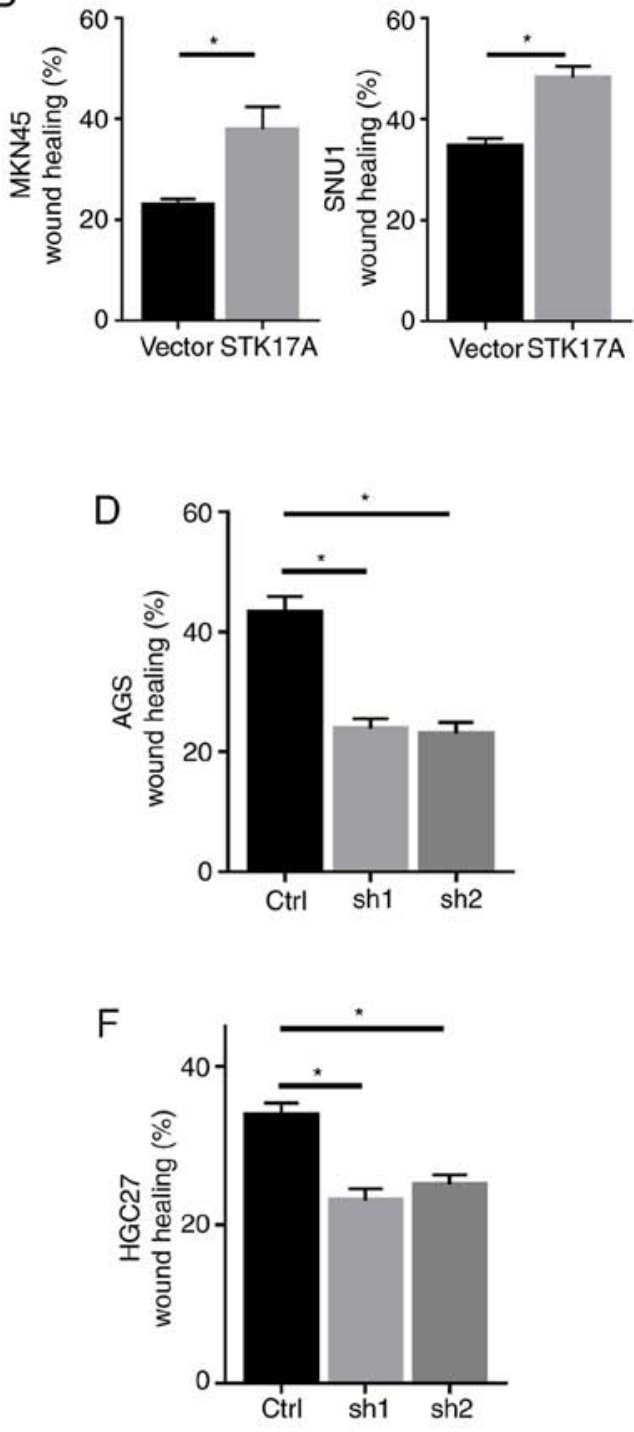

$\mathrm{H}$

Ctrl

sh1

sh2
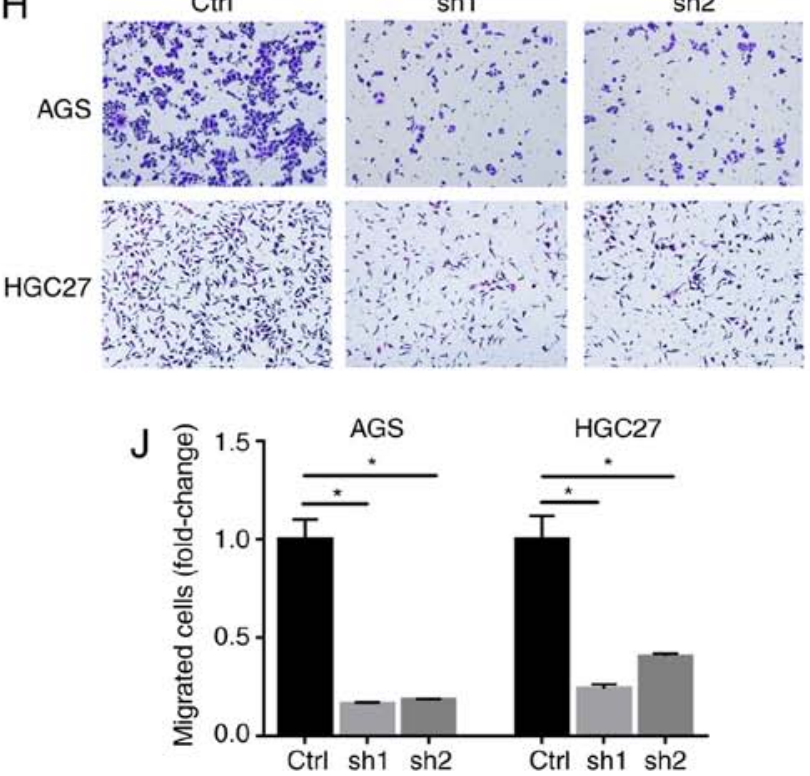

Figure 4. STK17A downregulation inhibits GC cell proliferation and migration. (A and B) The effects of STK17A upregulation on the migratory capability of GC cells using the wound-healing assay (Olympus CKX 53 light microscope, $\mathrm{x} 40$ ). (C-F) The effects of STK17A downregulation on the migratory capability of GC cells using the wound-healing assay (Olympus CKX 53 light microscope; magnification x40). The effects of STK17A (G and I) upregulation and ( $\mathrm{H}$ and $\mathrm{J}$ ) downregulation on the migratory capabilities of GC cells using the Transwell assay (Olympus BX 53 light microscope; magnification; $\mathrm{x} 100$ ). "P<0.05. STK17A, serine/threonine kinase 17a; GC, gastric cancer; Ctrl, control; sh, short haiprin. 

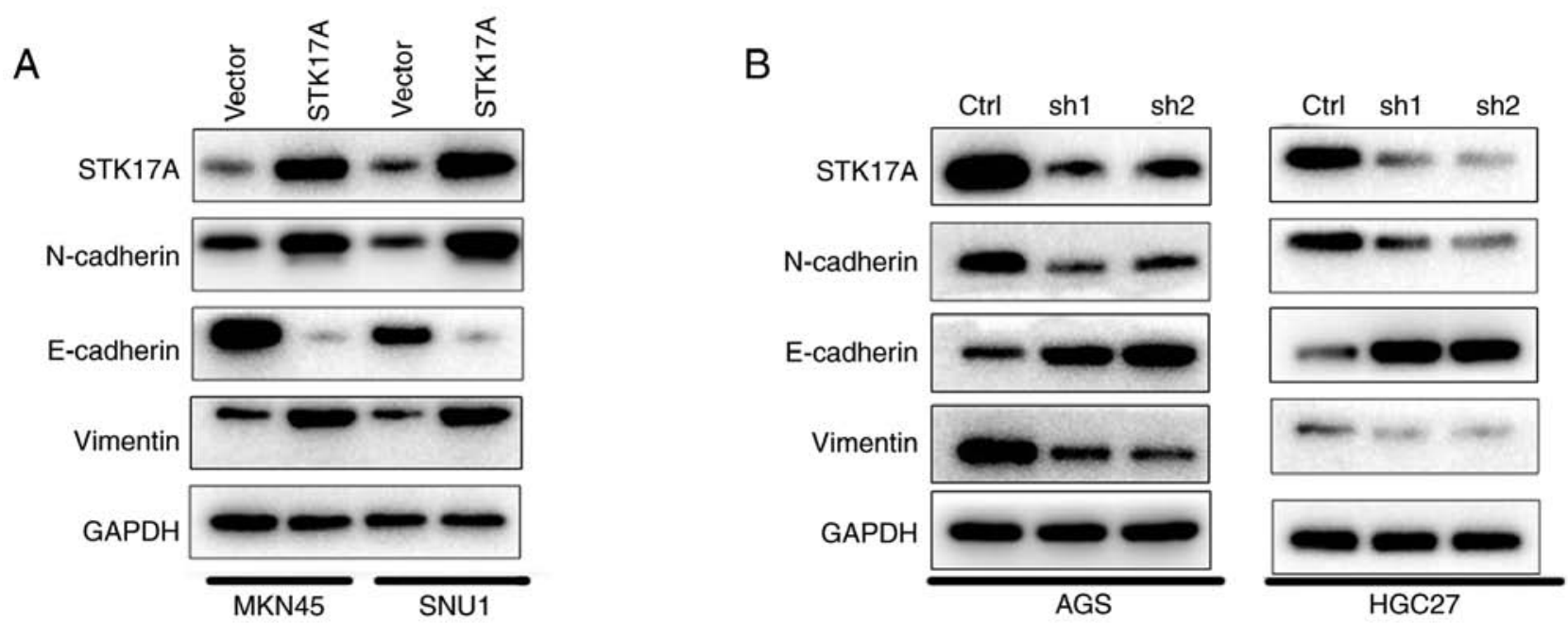

Figure 5. (A and B) Western blot analyses of the expression levels of STK17A, E-cadherin, N-cadherin, and vimentin in the STK17A downregulation and upregulation cell lines. STK17A, serine/threonine kinase 17a; Ctrl, control; sh, short haiprin.

\section{Results}

STK17A is upregulated in GC. Of the $39 \mathrm{GC}$ tissue specimens, $31(79.5 \%)$ exhibited higher STK17A mRNA expression compared with their paired adjacent specimens $(\mathrm{P}=0.0009$; Fig. 1A). There was also a significant difference in STK17A protein levels between $\mathrm{GC}$ and adjacent normal tissues $(\mathrm{P}<0.001$; Fig. 1B and C). Furthermore, STK17A expression was markedly upregulated in all six GC cell lines detected compared to GES1 by PCR (Fig. 1D) and western blotting (Fig. 1E).

Upregulation of STK17A is associated with advanced clinicopathological characteristics of GC. The associations between STK17A expression and the clinicopathological characteristics of GC are presented in Table II. The analysis results demonstrated that the overexpression of the STK17A protein was significantly associated with Lauren classification $(\mathrm{P}=0.018)$, pTNM stage $(\mathrm{P}<0.001)$, tumor invasion depth $(\mathrm{P}<0.001)$, lymph node metastasis $(\mathrm{P}<0.001)$ and 5-year survival $(\mathrm{P}<0.001)$. No other clinicopathological parameters, such as patient sex $(\mathrm{P}=0.815)$, age $(\mathrm{P}=0.695)$, tumor location $(\mathrm{P}=0.449)$ or differentiation grade $(\mathrm{P}=0.384)$. were found to be associated with STK17A expression levels.

High expression of STK17A in GC tissues is associated with poor patient survival. The study demonstrated that pTNM stage, lymph node metastasis, depth of tumor invasion and STK17A protein expression levels were prognostic factors for overall survival (OS) and progression-free survival (PFS) in GC (Table III). Multivariate analysis further indicated that STK17A protein expression and pTNM stage were independent risk factors for OS and PFS (Table IV). The OS (Fig. 2A) and PFS (Fig. 2B) analyses revealed that patients with high expression of STK17A exhibited shorter survival time compared with patients exhibiting normal STK17A expression. Furthermore, statistically significant differences in OS were found between STK17A expression in patients with $\mathrm{pTNM}$ stage I-II $(\mathrm{P}=0.019)$ and those with pTNM stage III-IV ( $<<0.001$; Fig. $2 \mathrm{C}$ and D).
Upregulation of STK17A promotes the proliferation of GC cells. As revealed in Fig. 3, to investigate the functional effects of STK17A, STK17A was overexpressed in MKN45 and SNU1 GC cells (Fig. 3A). CCK-8 assays revealed that overexpression of STK17A significantly promoted the proliferation of GC cells (Fig. 3B and C). Compared to vector-transfected cells, overexpression of STK17A also significantly increased the mean number of colonies in the colony formation assay (Fig. 3G-I). In parallel, STK17A-knockdown stable cell lines were established in AGS and HGC27 cells (Fig. 3D). CCK-8 and colony formation assays both indicated that knockdown of STK17A significantly reduced the proliferation of GC cells (Fig. 3E and F and J-L). These results suggested that STK17A could promote the proliferation of GC cells.

STK17A regulates the migration of GC cells via epithelialmesenchymal transition (EMT). The effect of STK17A on the migration ability of GC cells was further evaluated. In the wound healing assay, MKN45 and SNU1 cells overexpressing STK17A exhibited more rapid wound closure compared with vector-expressing cells (Fig. 4A and B). Conversely, AGS and HGC27 cells with STK17A knockdown exhibited slower wound closure (Fig. 4C-F). Consistently, similar results were obtained with the Transwell assay. STK17A overexpression significantly enhanced the migration ability of MKN45 and SNU1 cells (Fig. 4G), while STK17A knockdown in AGS and HGC27 cells suppressed their migration ability (Fig. 4H). Consistent with the aforementioned results, the western blot analysis results demonstrated that STK17A overexpression significantly increased the expression of $\mathrm{N}$-cadherin and vimentin, but inhibited the expression of E-cadherin (Fig. 5A), while STK17A downregulation produced the opposite results, as predicted (Fig. 5B).

\section{Discussion}

With the continuous development of clinical diagnosis and treatment technology, the clinical prognosis of patients with GC has significantly improved. However, patients with the 
similar clinicopathological characteristics exhibit different clinical results when receiving the same therapy. Therefore, it is imperative to identify new biomarkers, which may accurately predict the prognosis of patients and develop individualized treatments for patients with GC.

The present study demonstrated that STK17A was upregulated at both the mRNA and protein levels in GC compared with adjacent normal gastric tissues. It was observed that the STK17A expression level was correlated with clinical and pathological factors, specifically tumor invasion depth and lymph node metastasis. On clinical outcome analysis, patients with STK17A overexpression exhibited significantly shorter survival and poorer prognosis. These data provided evidence that STK17A expression may be associated with the malignant biological behavior of GC, clinicopathological characteristics and prognosis of GC. Therefore, STK17A may be considered as a potential prognostic biomarker. It was demonstrated that GC patients with lymph node metastasis and greater depth of tumor invasion exhibited higher expression levels of STK17A, and these results suggested that STK17A may promote GC growth and metastasis. Furthermore, it was demonstrated through functional analysis that high STK17A expression enhanced the proliferation and migration of GC cells. In addition, mechanistic studies demonstrated that STK17A promoted GC migration via mediating EMT.

EMT has been reported to be an important process in tumor occurrence and metastasis. EMT is characterized by loss of epithelial marker expression, increased mesenchymal marker expression, and enhanced migratory and invasive cell behaviors (24). Consistently, in the present study, upregulation of STK17A inhibited the expression of the epithelial marker E-cadherin, while it enhanced the expression of the mesenchymal markers $\mathrm{N}$-cadherin and vimentin. Furthermore, STK17A was shown to promote tumor cell invasion and motility by changing the cell-cell and cell-stroma interactions (25). It has been reported that EMT of GC cells may affect the pathogenesis of GC through the Wnt/ $\beta$-catenin, PI3K/AKT, and HGF/c-Met signaling pathways, which provides an important approach to the study of the relationship between cell behavior and EMT (26-28).

It was previously demonstrated that, in some tumor types, STK17A is considered to act as a tumor promoter: STK17A was revealed to promote glioblastoma cell proliferation, and its overexpression may be considered as a biomarker for advanced cervical cancer $(8,29)$. However, it was also demonstrated that STK17A overexpression reduced cell proliferation in testicular and ovarian cancer (7). These results support the highly relevant specificity and dependence of STK17A function on cancer type. These effects may be closely associated with the p53-dependent signaling mechanism, as the study revealed that STK17A is one of the direct and DNA damage-inducible p53 target genes. Moreover, STK17A has functional and shared response elements upstream of the p53 pathway, which is involved in cell signal transduction $(7,30)$. These findings provide new insight into the mechanism through which STK17A may promote GC cell proliferation.

There remains a limitation in this research. It has been demonstrated that STK17A has a close relationship with chemoresistance in ovarian cancer cells (6). Unfortunately, the association between STK17A and chemoresistance in GC has not been fully evaluated. This issue may be addressed in a future study.

In summary, STK17A was revealed to be upregulated in GC tissues in the present study, and high STK17A expression was significantly associated with clinical metastasis and poor prognosis in patients with GC. In addition, STK17A may promote cell proliferation and migration via regulation of EMT, thereby providing a potential therapeutic target for GC.

\section{Acknowledgements}

Not applicable.

\section{Funding}

The present study was supported by the National Natural Science Foundation of China (grant no. 81872264).

\section{Availability of data and materials}

The datasets used and/or analyzed during the current study are available from the corresponding author on reasonable request.

\section{Authors' contributions}

ZW, YQ and YJ conceived and designed the study. ZW, CW, LL and JQ performed the experiments and analyzed the data. LS, SL and LW collected the specimens and performed patient follow-up. ZW and CW wrote the paper. YQ, BJ and YJ reviewed the manuscript for important intellectual content and edited the manuscript. ZW and CW performed the experiments required for revision. All authors read and approved the manuscript and agree to be accountable for all aspects of the research in ensuring that the accuracy or integrity of any part of the work are appropriately investigated and resolved.

\section{Ethics approval and consent to participate}

Written informed consent for the use of tissue samples was obtained from all patients or their legal guardians. The use of GC tissues by the present study was approved by the Ethics Committee of Zhengzhou University.

\section{Patient consent for publication}

Not applicable.

\section{Competing interests}

The authors declare that they have no competing interests.

\section{References}

1. Sung H, Ferlay J, Siegel R, Laversanne M, Soerjomataram I, Jemal A and Bray F: Global cancer statistics 2020: GLOBOCAN estimates of incidence and mortality worldwide for 36 cancers in 185 countries. CA Cancer J Clin: Feb 4, 2021 (Online ahead of print).

2. Cidon EU, Ellis SG, Inam Y, Adeleke S, Zarif S and Geldart T: Molecular targeted agents for gastric cancer: A step forward towards personalized therapy. Cancers (Basel) 5: 64-91, 2013. 
3. Steeg PS: Metastasis suppressors alter the signal transduction of cancer cells. Nat Rev Cancer 3: 55-63, 2003.

4. Sanjo H, Kawai T and Akira S: DRAKs, novel serine/threonine kinases related to death-associated protein kinase that trigger apoptosis. J Biol Chem 273: 29066-29071, 1998.

5. Bialik S and Kimchi A: The death-associated protein kinases: Structure, function, and beyond. Annu Rev Biochem 75: 189-210, 2006.

6. Gao J, Liu D, Li J, Song Q and Wang Q: Effect of STK17A on the sensitivity of ovarian cancer cells to paclitaxel and carboplatin. Oncol Lett 12: 1107-1112, 2016.

7. Mao P, Hever MP, Niemaszyk LM, Haghkerdar JM, Yanco EG Desai D, Beyrouthy MJ, Kerley-Hamilton JS, Freemantle SJ and Spinella MJ: Serine/threonine kinase 17A is a novel p53 target gene and modulator of cisplatin toxicity and reactive oxygen species in testicular cancer cells. J Biol Chem 286: 19381-19391, 2011.

8. Mao P, Hever-Jardine MP, Rahme GJ, Yang E, Tam J, Kodali A, Biswal B, Fadul CE, Gaur A, Israel MA and Spinella MJ: Serine/threonine kinase 17A is a novel candidate for therapeutic targeting in glioblastoma. PLoS One 8: e81803, 2013.

9. Park Y, Kim W, Lee JM, Park J, Cho JK, Pang K, Lee J, Kim D, Park SW, Yang KM and Kim SJ: Cytoplasmic DRAK1 overexpressed in head and neck cancers inhibits TGF- $\beta 1$ tumor suppressor activity by binding to $\mathrm{Smad} 3$ to interrupt its complex formation with Smad4. Oncogene 34: 5037-5045, 2015.

10. Short SP, Thompson JJ, Bilotta AJ, Chen X, Revetta FL, Washington MK and Williams CS: Serine threonine kinase 17A maintains the epithelial state in colorectal cancer cells. Mol Cancer Res 17: 882-894, 2019

11. Wei W and Liu C: Prognostic and predictive roles of microRNA-411 and its target STK17A in evaluating radiotherapy efficacy and their effects on cell migration and invasion via the p53 signaling pathway in cervical cancer. Mol Med Rep 21: 267-281, 2020.

12. Temmerman K, Simon B and Wilmanns M: Structural and functional diversity in the activity and regulation of DAPK-related protein kinases. FEBS J 280: 5533-5350, 2013.

13. Wang WJ, Kuo JC, Yao CC and Chen RH: DAP-kinase induces apoptosis by suppressing integrin activity and disrupting matrix survival signals. J Cell Biol 159: 169-179, 2002.

14. Lin Y, Hupp TR and Stevens C: Death-associated protein kinase (DAPK) and signal transduction: Additional roles beyond cell death. FEBS J 277: 48-57, 2010.

15. Kiger AA, Baum B, Jones S, Jones MR, Coulson A, Echeverri C and Perrimon N: A functional genomic analysis of cell morphology using RNA interference. J Biol 2: 27, 2003.

16. Neubueser D and Hipfner DR: Overlapping roles of Drosophila Drak and Rok kinases in epithelial tissue morphogenesis. Mol Biol Cell 21: 2869-2879, 2010.

17. Robertson F, Pinal N, Fichelson P and Pichaud F: Atonal and EGFR signalling orchestrate rok- and Drak-dependent adherens junction remodelling during ommatidia morphogenesis Development 139: 3432-3441, 2012.
18. Coleman ML, Sahai EA, Yeo M, Bosch M, Dewar A and Olson MF: Membrane blebbing during apoptosis results from caspase-mediated activation of ROCK I. Nat Cell Biol 3: 339-345, 2001.

19. Gilmore AP. Anoikis. Cell Death Differ 12 (Suppl 2): S1473-S1477, 2005.

20. Tang H, Liu YJ, Liu M and Li X: Establishment and gene analysis of an oxaliplatin-resistant colon cancer cell line THC8307/ L-OHP. Anticancer Drugs 18: 633-639, 2007.

21. Edge SB, Byrd DR, Compton CC, Fritz AG, Greene FL and Trotti A (eds). AJCC cancer staging manual. 7th edition. New York, NY, Springer, 2010.

22. Livak KJ and Schmittgen TD: Analysis of relative gene expression data using real-time quantitative PCR and the 2(-Delta Delta C(T)) method. Methods 25: 402-408, 2001.

23. Li Y, Nie CJ, Hu L, Qin Y, Liu HB, Zeng TT, Chen L, Fu L, Deng W, Chen SP, et al: Characterization of a novel mechanism of genomic instability involving the SEI1/SET/NM23H1 pathway in esophageal cancers. Cancer Res 70: 5695-5705, 2010.

24. Kalluri R and Weinberg RA: The basics of epithelialmesenchymal transition. J Clin Invest 119: 1420-1428, 2009.

25. Inoue T, Umezawa A, Takenaka T, Suzuki H and Okada H: The contribution of epithelial-mesenchymal transition to renal fibrosis differs among kidney disease models. Kidney Int 87: 233-238, 2015

26. Chen DH, Yu JW and Jiang BJ: Contactin 1: A potential therapeutic target and biomarker in gastric cancer. World J Gastroenterol 21: 9707-9716, 2015.

27. Lamouille S, Xu J and Derynck R: Molecular mechanisms of epithelial-mesenchymal transition. Nat Rev Mol Cell Biol 15: 178-196, 2014.

28. Mesteri I, Schoppmann SF, Preusser M and Birner P: Overexpression of CMET is associated with signal transducer and activator of transcription 3 activation and diminished prognosis in oesophageal adenocarcinoma but not in squamous cell carcinoma. Eur J Cancer 50: 1354-1360, 2014.

29. Thomas A, Mahantshetty U, Kannan S, Deodhar K, Shrivastava SK, Kumar-Sinha C and Mulherkar R: Expression profiling of cervical cancers in Indian women at different stages to identify gene signatures during progression of the disease. Cancer Med 2: 836-648, 2013.

30. Cekirge HS, Peynircioglu B and Saatci I: Endovascular treatment of an 'anterior cerebral artery' aneurysm in a patient with 'embryonic unfused middle cerebral artery' anomaly: A case report. Neuroradiology 47: 690-694, 2005.

(i) (9) This work is licensed under a Creative Commons Attribution-NonCommercial-NoDerivatives 4.0 International (CC BY-NC-ND 4.0) License. 